\title{
Servicios ambientales: sistema agroforestal tradicional con plantas de maguey pulquero en la Altiplanicie, Hidalgo
}

\author{
Alberto Ulises Narváez Suáres ${ }^{1}$ \\ Artemio Cruz León ${ }^{1 \S}$ \\ Dora Ma. Sangerman-Jarquín ${ }^{2}$ \\ ${ }^{1}$ Ciencias en Desarrollo Rural Regional-Universidad Autónoma Chapingo. Carretera México-Texcoco km \\ 38.5, Chapingo, Texcoco, Estado de México. (aunarvaez@ hotmail.com). ${ }^{2}$ Campo Experimental Valle de \\ México-INIFAP. Carretera Los Reyes-Texcoco km 13.5, Coatlichán, Texcoco, Estado de México. CP. \\ 56250. Tel. 80008822 22, ext. 85353. (sangerman.dora@infap.gob.mx). \\ ${ }^{\S}$ Autor para correspondencia: acruzl@chapingo.mx.
}

\section{Resumen}

Los sistemas agroforestales tradicionales en México son una expresión de las formas de manejo y aprovechamiento de recursos naturales con una antigüedad más allá de la llegada de los europeos, la obtención de servicios ambientales y cosecha múltiples en periodos prolongados son característica importante para destacar. La región de la Altipanicie pulquera en el estado de Hidalgo posee las condiciones y práctica del sistema agroforestal con maguey pulquero como planta perenne principal al que se pueden asociar las prácticas de manejo para la obtención de servicios ambientales diversos y variados productos satisfactores de necesidades humanas. Por ello, este sistema puede ayudar a resolver los problemas ambientales en la región y apoyar las economías locales de los productores campesinos.

Palabras clave: agroforestería, conservación de suelo, cosecha de agua, cosecha múltiple, estrategia socieconómica.

Recibido: octubre de 2020

Aceptado: diciembre de 2020 
Actualmente este sistema de producción capitalista 'devora $25 \%$ más recursos de los que la naturaleza puede reponer' (Bartra, 2009), ¿qué sería de este planeta si todos los países del mundo estuvieran en las mismas condiciones económicas y sobreexplotaran la naturaleza al mismo ritmo de los países 'desarrollados'? El panorama actual sería catastrófico, tal vez, el fin de la civilización tal como la conocemos.

La gran crisis es sistémica. Esta tiene diferentes manifestaciones: crisis medioambiental, energética, alimentaria, migratoria, bélica y económica. En su conjunto cada una de sus manifestaciones está llevando al sistema a un colapso que tiene alcances mundiales. Se tiene un capitalismo del fin del mundo (Bartra, 2017).

Pero de este conjunto, es la crisis medioambiental la que está poniendo en duda la continuidad de la vida humana sobre el planeta. Esta crisis, se trata de una devastación ecológica múltiple, que incluye las alteraciones en los ciclos del nitrógeno y el fósforo, la contaminación del agua, los efectos de los aerosoles sobre la atmósfera, la polución química, el agotamiento del ozono estratosférico y la acidificación de los océanos, procesos que, reunidos juntos, configuran la sexta extinción masiva de la biodiversidad (Giraldo, 2014).

La revolución verde como expresión del intento de emular la revolución industrial, busca producir alimentos a bajo costo, además de aprovechar los subsidios de las metrópolis. Esta producción tiene a un alto costo energético, emplea de energía fósil, agroquímicos y trabajo humano proveniente de países subdesarrollados, adicionalmente del agua extraída de estos mismos países quienes también sufren la contaminación y degradación de sus tierras bajo el modelo agroexportador capitalista. A nivel global se estima que $86 \%$ de la huella hídrica está relacionada con el consumo de productos agrícolas, $10 \%$ con el consumo de bienes industriales y menos de $5 \%$ con los usos domésticos (Delgado, 2014).

Frente a esta situación es urgente incrementar la producción de alimentos de manera sustentable, dejar de lado el paradigma de la revolución verde y retomar técnicas de producción de los pueblos nativos que resultan menos dañinas al ambiente y que permitieron la subsistencia y desarrollo de la civilización desde el invento de la agricultura hace más de 10 mil años. El reconocimiento y fomento de sistemas agroforestales para la producción de alimentos es de alto potencial como alternativa sustentable, es el objetivo del presente documento ejemplificado con la producción de cultivos anuales y maguey pulquero.

\section{Región de estudio y caracterización}

Históricamente el estado de Hidalgo se ha caracterizado por ser un estado pobre y con alta desigualdad social a pesar de las riquezas naturales con que cuenta: hoy en día, para la Comisión Económica para América Latina y el Caribe (CEPAL), la estructura productiva hidalguense es territorio no dinámico y con bajo producto interno bruto (PIB) per cápita, crece por debajo de la media del país y sus productos per cápita están también por debajo del promedio nacional (Roldan, 2015).

En lo que se refiere a su ubicación espacial Hidalgo está ubicado parcialmente en la Cuenca de México y la región montañosa de la Cordillera Neo-Volcánica, la cual divide la entidad en tres grandes regiones naturales: 1) la planicie, localizada al sur y la de mayor acceso a la Ciudad de 
México y a la región centro; 2) la serranía, en el macizo montañoso que cruza la entidad; y 3) la Huasteca, localizada al norte, que colinda con San Luis Potosí y Veracruz (Roldan, 2015). La Altiplanicie pulquera hidalguense forma parte de la primera región natural mencionada y es precisamente esa cercanía con la Ciudad de México y otros estados del centro una de sus fortalezas para desarrollarse con recursos propios, en este caso el maguey pulquero y la gran cantidad de subproductos que de esta planta se derivan.

Esta regionalización agrupa a los 84 municipios del estado de Hidalgo en diez regiones geográficoculturales: la Huasteca, las sierras Alta, Baja, Gorda y la de Tenango, el Valle de Tulancingo, la Comarca Minera, la Altiplanicie pulquera, la Cuenca de México y el Valle del Mezquital (Roldán, 2017) (Figura 1).

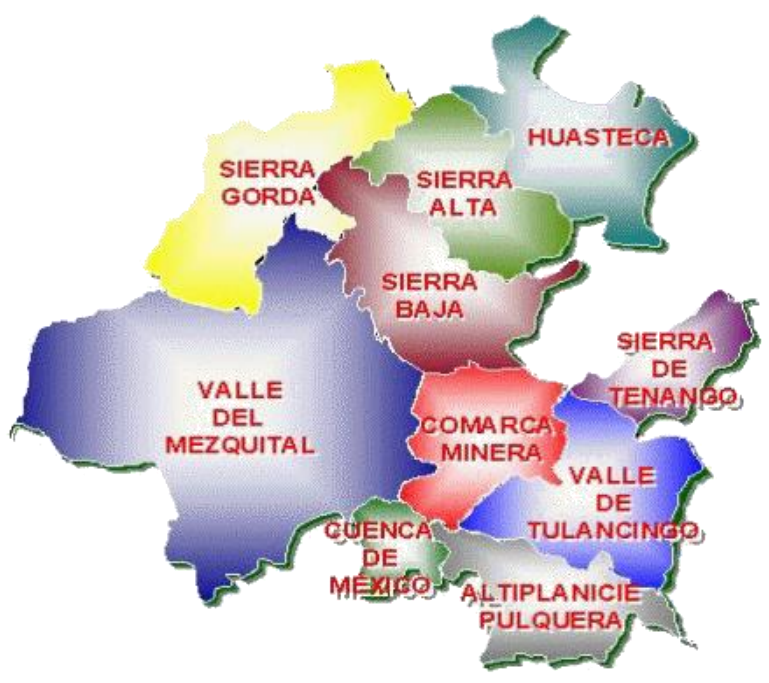

Figura 1. Regiones del estado de Hidalgo. http://www.inafed.gob.mx/work/enciclopedia/ EMM13hidalgo/regionalizacion.html.

\section{Altiplanicie pulquera}

Conformada por los municipios de Apan, Almoloya, Emiliano Zapata, Tepeapulco, Tlanalapa, Zempoala, Epazoyucan y Singuilucan, el clima de la Altiplanicie es motivo suficiente para no sembrar más que magueyes y cebada, porque éstas son de las pocas plantas que resisten las frecuentes heladas que caen en la zona, sobre todo en invierno cuando el termómetro suele precipitarse varios grados debajo de cero, pero con la ausencia de nieve (INAFED, 2018).

\section{¿Qué es un sistema agroforestal?}

El International Council for Research in Agroforestry (1983) lo define como: sistema sostenido del manejo de la tierra que aumenta su rendimiento total, combina la producción de cultivos con especies forestales y animales, en forma simultánea o secuencial sobre la misma superficie de terreno y aplica prácticas de manejo que son compatibles con las prácticas culturales de la población local (Iglesias, 1999). 
El funcionamiento de estos sistemas en una parcela de temporal conlleva varios beneficios como: producción de madera, mejoramiento del microclima y el suelo, aporte de materia orgánica, fijación de nitrógeno y captación de dióxido de carbono, producción de follaje y otros productos alimenticios y medicinales, gomas, resinas y fibras (Iglesias, 1999). Un sistema agroforestal con agave pulquero eleva la productividad de las parcelas, sus beneficios son permanentes y la aplicación de insumos externos es mínima y económicamente están al alcance del productor.

Una plantación de maguey pulquero incremente su producción con la aplicación de estiércol procesado, producto local al alcance de los productores de la Altiplanicie pulquera hidalguense, debido a la combinación de la actividad agrícola con la pecuaria. Los rebaños de ovinos y caprinos, aportan la materia prima para la fertilización, además de la carne para satisfacer la demanda de barbacoa que tiene la región.

\section{Servicios ambientales de los sistemas agroforestales}

Se tiene conocimiento que los sistemas agroforestales (SAF) tienen mayor ventaja que los monocultivos convencionales para proporcionar servicios ambientales, característica distintiva por su origen mesoamericano, que han sido utilizados desde tiempo ancestral y que hoy siguen funcionando para proporcionar productos básicos que satisfacen las necesidades de las familias. Algunos de los principales servicios ambientales que provee un sistema agroforestal en las parcelas y al medio ambiente en general son: 1) mantenimiento de la fertilidad del suelo/reducción de la erosión mediante el aporte de material orgánico al suelo, fijación de nitrógeno y reciclaje de nutrientes; 2) conservación del agua (cantidad y calidad) al favorecer la infiltración y reducir la escorrentía superficial que podría contaminar cursos de agua; 3 ) captura de carbono, enfatizando el potencial de los sistemas silvopastoriles; y 4) conservación de la biodiversidad en paisajes fragmentados. Estos servicios complementan los productos que los SAF proveen (para uso comercial o familiar; por ejemplo, leña, madera, frutos) (Beer, 2003).

El maguey pulquero, y en general todos los agaves son considerados por la ley como un recurso forestal no maderable, la norma oficial mexicana NOM-007-REC-NAT-1997 establece los procedimientos, criterios y especificaciones para realizar el aprovechamiento, transporte y almacenamiento de ramas, hojas o pencas, flores, frutos y semillas (DOF, 1997).

Taxonómicamente el género Agave se ubica en la familia Agavaceae. En el Continente Americano se reportan aproximadamente 310 especies, de las cuales en México existen 272, por ello se considera a este país como centro de origen del género (Granados, 1993). Son plantas adaptadas a condiciones de aridez. Raíces someras y ramificadas, cutícula gruesa, suculencia, estomas hundidos, metabolismo fotosintético y metabolismo ácido de crasuláceas (MAC), atributos que le permiten establecerse en zonas carentes de agua (Granados, 1993) y sobre estas características se basan sus ventajas ambientales.

La importancia económica de los magueyes se establece por los productos principales obtenidos, los grupos resultantes son magueyes: textileros, pulqueros y mezcaleros (Ruvalcaba, 1983). En algunas regiones de México cada uno de estos grupos ha sido la base de la identidad, cultura y economía, sobre su explotación han desarrollado la prosperidad de la región, motivo por el cual al sufrir cambios en la demanda de los productos derivados de algún grupo el auge económico han desarrollado o entrado en crisis estos territorios, como ejemplo está el caso del henequén en la 
Península de Yucatán y del pulque en los llanos de Apan, que abarca varios municipios del estado de Hidalgo, Tlaxcala y Estado de México y recientemente el agave tequilero en Jalisco y estados con denominación de origen.

\section{Retención y formación de suelo agrícola}

El maguey es eficiente para formar suelo agrícola, Ruvalcaba (1983) describe como en el municipio de Epazoyucan, en el estado de Hidalgo, y más específicamente en un rancho denominado. El techalote, el propietario logró recuperar entre 8 y 10 ha de terreno en tierras con pendiente a base de terrazas. Estas estructuras son parte del paisaje del altiplano y expresión de los sistemas agroforestales que desde la época prehispánica se establecen en la región, las terrazas son parte fundamental del manejo agrícola que capta, controla el agua de lluvia, además de permitir la recarga de los mantos freáticos. También permite la retención y control de arrastre de suelos que permite una fertilidad duradera.

Los productores agropecuarios de la región poseen los conocimientos y experiencia para el diseño y construcción de terrazas, así como aquellos necesarios para la plantación de los magueyes sobre los bordos de las terrazas, con lo que se logra terrazas de formación sucesiva. También en la región se han construido terrazas con maquinaria pesada, lo cual facilita el trabajo y requiere de apoyos de gobierno para dichas acciones.

De acuerdo con un estudio la región centro del país donde se ubican los estados de Tlaxcala, Puebla, Hidalgo, México y Querétaro que conforman la mayoría del Altiplano Central Mexicano presentan un alto porcentaje de degradación de sus suelos que alcanza 68\%, dentro del cual las causas principales son por degradación química e hídrica, seguida por degradación eólica y física (Moncada, 2013). Este proceso de degradación es el resultado de los cambios de cultivo de maíz a cebada maltera, cuyo paso de máquinas cosechadoras obliga a la eliminación parcial de terrazas, con lo cual se incrementa la erosión. El resultado ha sido la disminución de la importancia del maguey y por lo tanto su abandono, con lo cual se ha incrementado la erosión.

Los plantíos de maguey estaban regularmente distribuidos en los tiempos antiguos en líneas rectas de Sur a Norte y de Oriente a Poniente. Todos, o la mayor parte de los linderos de las propiedades estaban plantados con magueyes, y las tierras colgadas; es decir, en los declives de las montañas, se hallaban también abordadas con magueyes, para evitar que la tierra vegetal, arrastrada por las corrientes de las aguas, dejara estériles y desnudas las rocas, como ahora sucede en muchas fincas por el continuo corte de árboles, por la apatía y descuido o por la mal entendida economía de algunos hacendados. Indios fueron lo que descubrieron las propiedades de esta planta y la cultivaron, indios son los que hoy la cultivan, trasplantan y benefician (Rivera, 1990).

\section{Captación y control de agua de lluvia}

El terraceado es una práctica muy frecuente, siguiendo con el ejemplo anterior (Ruvalcaba, 1983) explica como el mismo productor aprovechó los bordos construidos a base de maguey para retener el agua de lluvia, para ello el productor desvió las corrientes de agua en las partes altas para que esta fluyera hacia sus terrenos y ahí la distribuía a lo largo de toda la superficie cultivable, esta práctica es conocida como 'cosecha de agua'. Esta forma de manejo del agua es parte del sistema de producción de 'arrope de humedad, que permite adelantar hasta en mes y medio la siembra para protegerse contra posibles heladas y granizadas tempranas, en la época de llenado de grano’. 


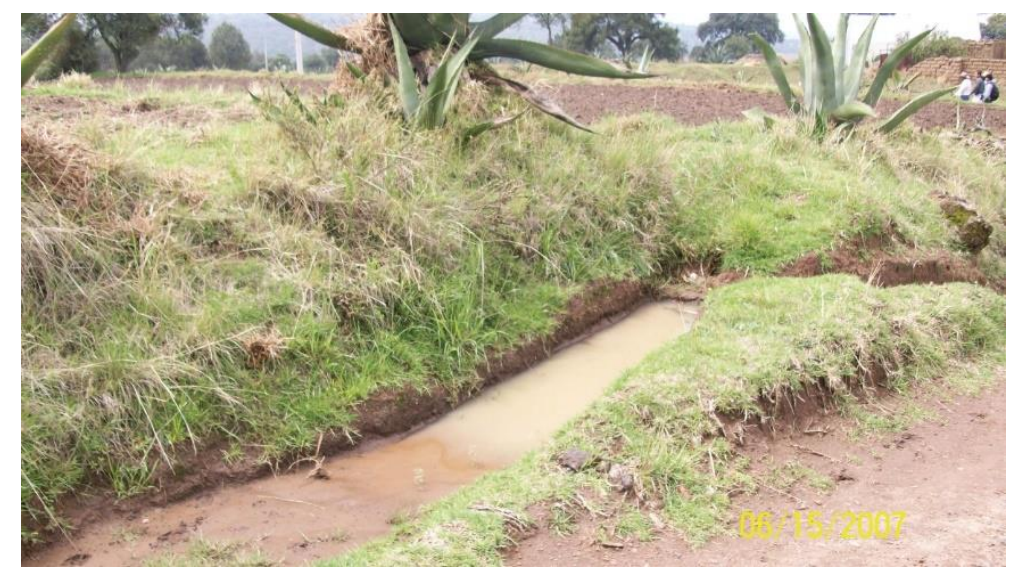

Figura 2. Zanjones para cosecha agua de lluvia en la comunidad de Álvaro obregón, Españita, Tlaxcala.

\section{Recarga de mantos freáticos}

El maguey es una planta que por su sistema radicular y morfología aprovecha eficientemente el agua y el manejo en terrazas y zanjas contribuye a retener agua y alimentar los mantos freáticos. Por la cantidad de biomasa almacena agua, una planta de maguey pulquero adulta puede pesar de $800 \mathrm{~kg}$ hasta $1500 \mathrm{~kg}$ dependiendo de la variedad. El manejo que se hace del maguey pulquero tiene capacidad para retener el agua y con ello permitir la recarga de los mantos freáticos, por ello diferentes dependencias buscan implementar proyectos de maguey en zonas con suelos pobres y climas áridos para reforestar y aprovechar así los múltiples beneficios ambientales de los agaves. El especialista de la CONAFOR resaltó que con estos apoyos que ofrece la dependencia federal, primeramente, es el captar agua, de ahí que se llevan a cabo este tipo de obras porque requiere, primero, recargar los mantos acuíferos (Notimex, 2018).

\section{Conformación de barreras vivas}

El maguey no solo es útil para delimitar terrenos, sino que constituye por sí mismo una cerca viva que protege de intrusos, de los vientos, además se genera un microclima que favorece la retención de humedad y suelo. Normalmente entre magueyes se agregan árboles frutales, nopal y otras especies de árboles o plantas de interés a los campesinos, favorecen la retención de suelo y aprovechamiento y control de agua de lluvia, conforman barreras vivas de gran calidad que favorecen la productividad de los cultivos y son fuente de alimentos que complementan la dieta de los productores e incrementan la productividad y el valor económico de la parcela. En la (Figura 3) se observa cómo se intercala el maguey con árboles frutales en una parcela y con ello la captación de agua y formación de barreras vivas.

\section{Formación de bordos}

La práctica de bordos e hileras es una protección inmejorable contra la erosión y deslave de tierra. La distancia entre bordos en la parte inclinadas depende del contorno y la pendiente del terreno. En ellos se mantiene la orientación tradicional. A los bordos en su conjunto se les deja la inclinación que dicta la experiencia para que se depositen los azolves a lo largo de ella y, por otra parte, que la 
velocidad del agua corra con saturación y sin arrastres del suelo fértil (Ruvalcaba, 1983). En la (Figura 3) se observan bordos sembrados con maguey, frutales y zanjones para retención de agua. En la actualidad con ayuda de la tecnología se pueden diseñar terrazas de excelente calidad a base de planos topográficos y cálculos matemáticos; sin embargo, cabe destacar la experiencia acumulada de los productores que diseñan sus bordos y terrazas basándose en su experiencia. En México se maneja el terraceado desde la época prehispánica.

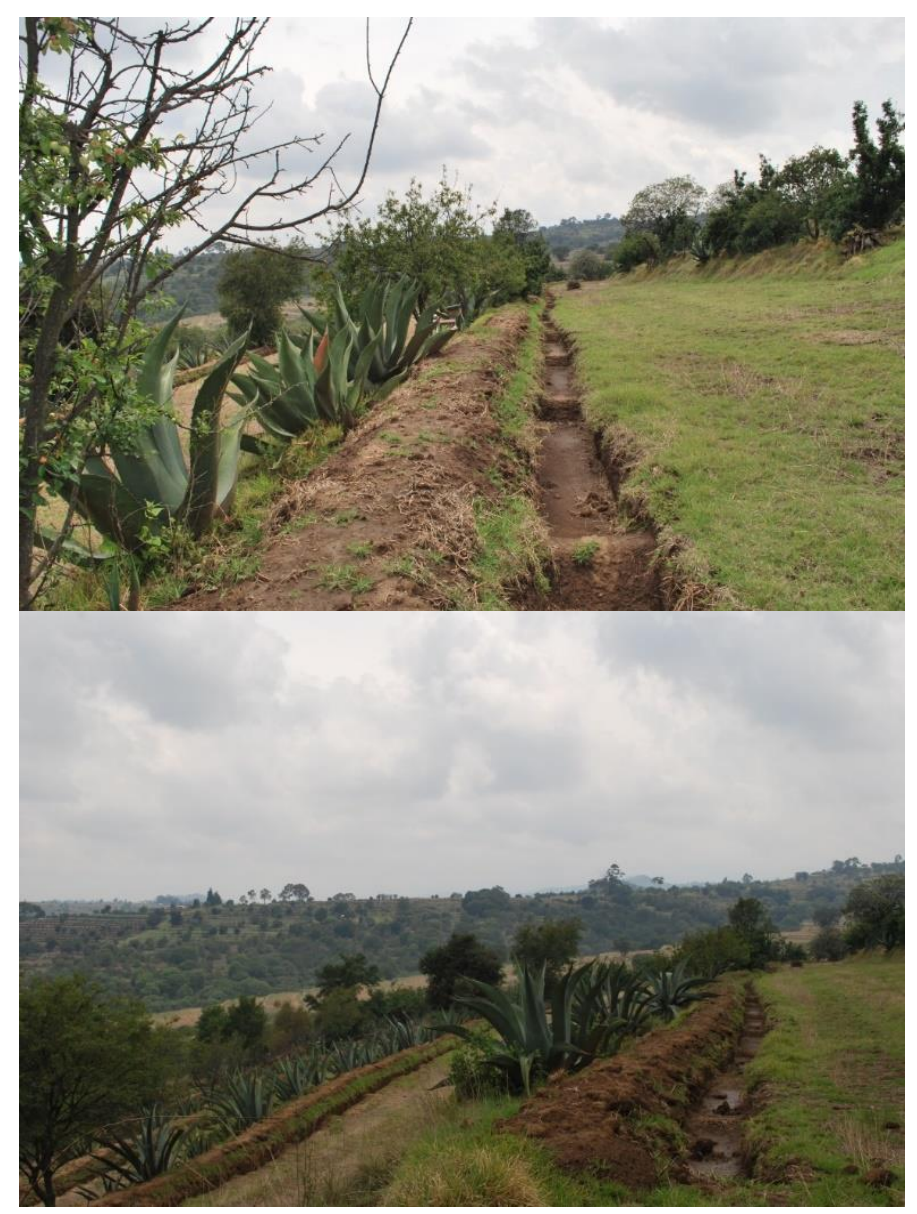

Figura 3. Terrazas conformadas con maguey pulque y árboles frutales.

\section{Conservación de la biodiversidad}

El maguey por sí solo es capaz de atraer animales e insectos que son benéficos para los cultivos, entre los animales que atrae son abejas, colibríes, avispas, palomillas, y murciélagos. Estos animales desempeñan un papel muy importante en la polinización y en el control de las poblaciones de insectos y algunos roedores. En el suelo adyacente a los magueyes podemos encontrar ratoncitos, cacomixtles, liebre, armadillo, tejón, tuza, víbora, lagartijas y hormigueros. Cada uno de estos animales desempeña un papel dentro de la cadena trófica del lugar, para la agricultura tienen importancia porque regulan la población de insectos y representan un control biológico de plagas. Si el maguey se intercala con nopal y árboles frutales la biodiversidad se incrementa y los servicios ecológicos que se prestan a los cultivos adyacentes se acentúan, a la par se incrementa los productos cosechados. 


\section{Aprovisionamiento de alimentos y remedios medicinales}

"Innumerables son los usos de esta planta. Toda entera sirve como leña y para cercar los campos; sus tallos se aprovechan como madera, sus hojas para cubrir los techos, como tejas, como platos, o fuentes, para hacer papiro, para hacer hilo con que se fabrican calzado, telas y toda clase de vestidos. Del jugo que mana y que destila en la cavidad media cortando los renuevos interiores $\mathrm{u}$ hojas más tiernas fabrican vinos, miel, vinagre y azúcar; dicho jugo provoca las reglas, ablanda el vientre, provoca la orina, limpia los riñones y la vejiga, rompe los cálculos y lava las vías urinarias... Las hojas asadas y aplicadas curan las convulsiones y calman los dolores... Esta planta por sí sola podría fácilmente proporcionar todo lo necesario para una vida frugal y sencilla, pues no la dañan los temporales ni los rigores del clima, ni la marchita la sequía. No hay otra cosa que dé mayor rendimiento (Hernández, 1959).

Además de todos los servicios ecológicos que el maguey pulquero proporciona y que ya fueron descritos están los múltiples beneficios que proporciona a las familias campesinas. Los productos de mayor valor comercial son el aguamiel, el pulque, los gusanos rojos y blanco, así como las pencas que se utilizan para la elaboración de barbacoa. Pero para una familia campesina el maguey tiene una amplia variedad de usos. Las pencas son utilizadas como recipientes para servir los alimentos en el campo durante el almuerzo y comida, también se utilizan para beber el pulque. Las púas se pueden utilizar como agujas para cerrar los costales. Los mezotes o pencas secas se utilizan como combustible para calentar los alimentos en la finca o en los hogares.

De las pencas y raíces se pueden obtener infinidad de remedios para aliviar enfermedades, también se puede extraer fibra para la elaboración de telas, canastos, escobas, cepillos y papel. El propio aguamiel y pulque poseen propiedades alimenticias y curativas, existen una gran cantidad de estudios al respecto donde se demuestra el contenido nutricional de estas bebidas que efectivamente poseen un gran valor alimenticio. Con ellas se pueden elaborar una gran cantidad de platillos como atoles, tamales y pan. El aguamiel deshidratado produce una miel de alto valor nutritivo que se puede utilizar para preparar mermeladas, galletas, atoles, agua de sabores y artículos para el cuidado de la piel.

El quiote, el huevo y las flores del maguey son base de varios platillos nutritivos y deliciosos que forman parte de la dieta de las familias campesinas. Los desechos del pulque se utilizan como un catalizador para la elaboración de composta que posteriormente se aplicará a los cultivos de maíz, frijol, calabaza, haba y trigo. El maguey pulquero es una planta útil para el campesino del altiplano central mexicano, su aprovechamiento es integral y es un excelente complemento de algunas necesidades de la familia. La Figura 4 muestra como los productores de la región intensifican la plantación de maguey pulquero de manera más intensiva combinándola con cultivos anuales como maíz, frijol, haba, alverjón y cebada. Algunos productores van cerrando cada vez más los espacios entre melgas. Estos cultivos aprovechan todos los beneficios de una cerca viva de maguey que ya se mencionaron anteriormente.

La forma más común es sembrarlo como lo ilustra la imagen es en hileras formando metepantle (Metl: maguey; Pantli: límite, hilera) aunque algunos productores de la región lo siembran en surco para aprovechas más el agua de lluvia (Figura 5). 


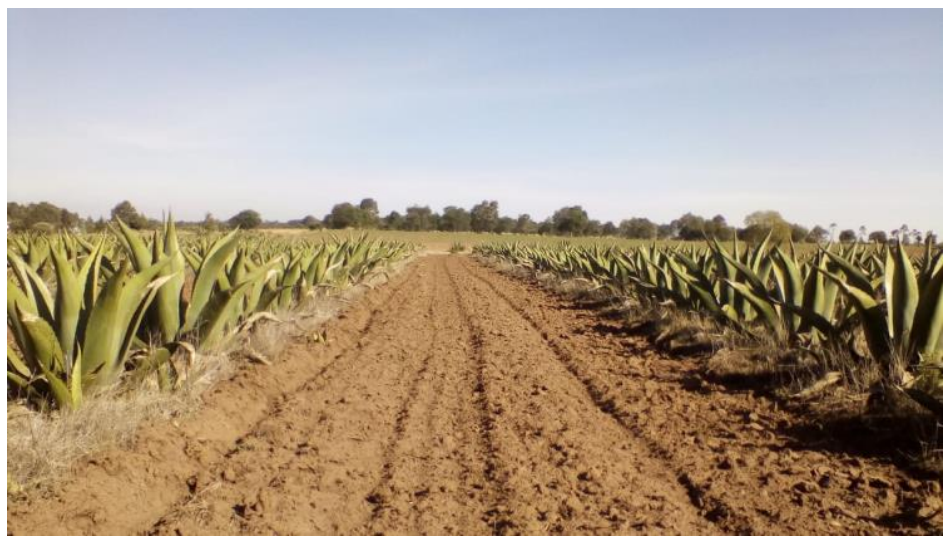

Figura 4. Plantación de agave pulquero combinado con cultivos anuales.

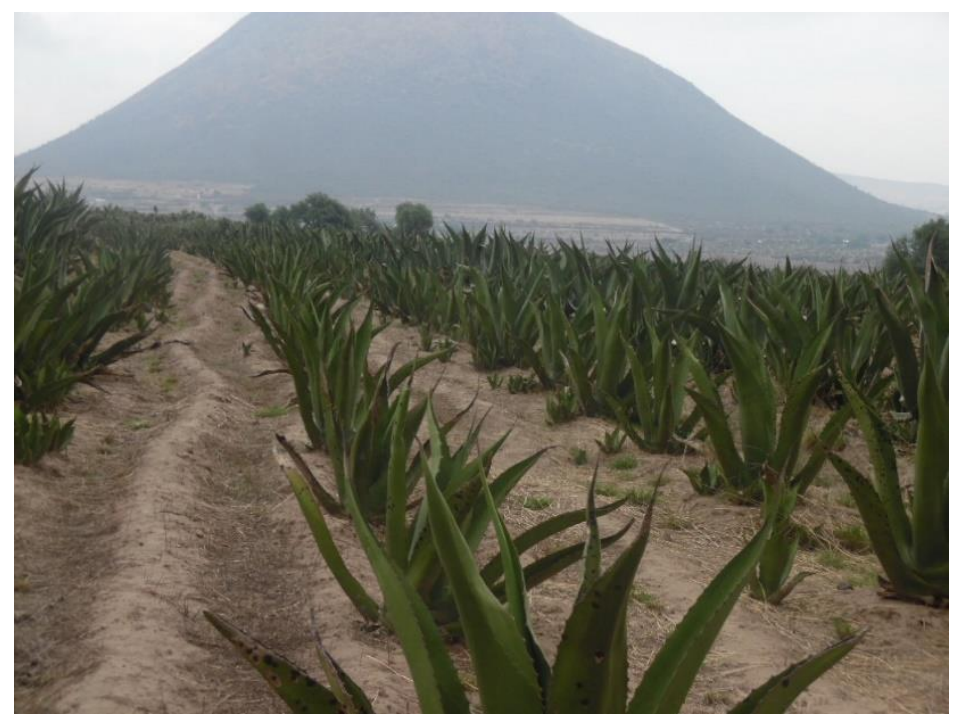

Figura 5. Plantación intensiva de maguey sembrado en surco, municipio de Zempoala, Hidalgo.

\section{Captación de bióxido de carbono}

Debido al tamaño de una planta adulta de maguey y la gran cantidad de biomasa que posee '... por sus características fisiológicas, los agaves son de las plantas que presentan mayor eficiencia en el uso del agua y en la captación de $\mathrm{CO}_{2}$ atmosférico que se conocen' (García, 2007). Por lo tanto, el agave pulquero es una planta que puede contribuir de manera importante a la captación de $\mathrm{CO}_{2}$ producto de la utilización de combustibles fósiles que contribuyen al calentamiento global. Además, es una planta que no es competitiva con otras especies por agua dada la cantidad mínima que requiere del líquido para su desarrollo. El metabolismo CAM le permite obtener ganancias netas de carbono con una pérdida mínima de agua (sifupro.org.mx).

\section{Servicios de turismo rural}

El turismo rural es una alternativa que ya es aprovechada por productores de maguey, utilizan su plantación, la arquitectura colonial junto con los productos derivados del maguey para ofrecer recorridos por las plantaciones, muestran el manejo del maguey, las variedades que manejan y 
ofrecen alimentos y bebidas a base de maguey, aguamiel y pulque. El turismo rural y cultural representa otra opción para los productores que pueden aprovechar las mismas plantaciones para atraer visitantes ya que la propia belleza de la planta, el recorrido por las magueyeras, su tradición y cultura son de interés para los habitantes de las zonas urbanas que deseen conocer un poco de esta tradición milenaria (Narváez, 2015).

\section{Ejemplo de un sistema agroforestal tradicional}

El agave y el maíz han sido fundamentales como base nutricional en la vida de los pueblos de México a lo largo de su historia (Figura 6).

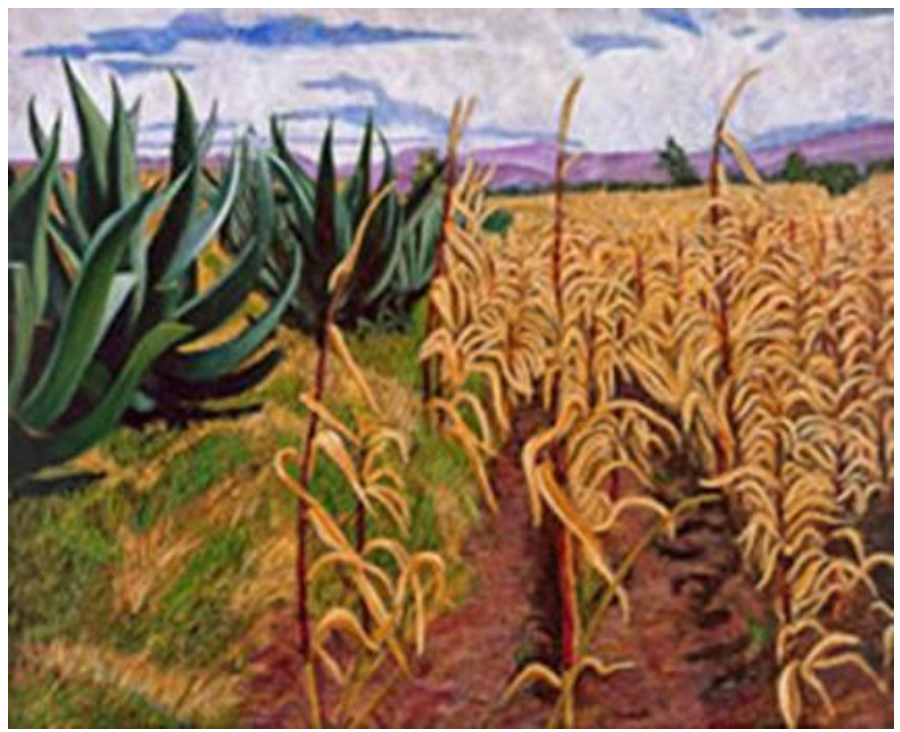

Figura 6. Maguey pulquero intercalado con maíz (http://www.pedrodiegoalvarado.com/obra_ paisaje.htm).

Esta forma de trabajar no ha variado entre las familias campesinas actuales, se utiliza por las ventajas que el maguey ofrece como barrera viva y la biodiversidad que atrae y con ello los beneficios a los cultivos anuales y perennes que se asocian con el maguey como los frutales. A pesar del abandono del maguey por casi 100 años, productores de subsistencia continúan utilizándolo para retener el suelo en zonas de pendiente. Se emplean en pendiente de 10 o 5 grados en tierras de temporal en las cuales el agua y el viento pueden remover el suelo. Esto se observó en todos los municipios que comprenden la región de la Altiplanicie pulquero se lleva a cabo la conservación de suelo y cuidado del maguey por ser conveniente para mantener sus tierras productivas como en los municipios de Epazoyucan, Zempoala, Tlanalapa, Singuilucan y demás.

En la Figura 7 se presentan los resultados de una encuesta de opinión sobre la situación actual del maguey pulquero en la región pulquera de hidalgo, en donde domina la percepción del abandono, olvido y extinción del cultivo. Hay una minoría que muestra resiliencia ante el problema de robo de mixiote, estos son los mixioteros, penqueros y chiniqüileros, que junto con su aprovechamiento encuentran formas de combatir el problema estableciendo vigilancia, penetración del meyolote y unión entre productores. Sin embargo, con los cuidados adecuados, de acuerdo con Narváez (2015) y sustentado por estudios de caso cuyo ejemplo es la empresa Pulcata, Puebla, se ha demostrado 
prácticamente que el maguey pulquero cultivado con los cuidados suficientes puede ser aprovechable para aguamiel a partir de los ocho años de plantado, antes se utilizan las penca para ximbo (platillo de pollo con nopales cocido en pencas pequeñas), hijuelos o incluso hormigueros de escamoles que se asocian muy bien con esta planta.

\section{Situación del cultivo}

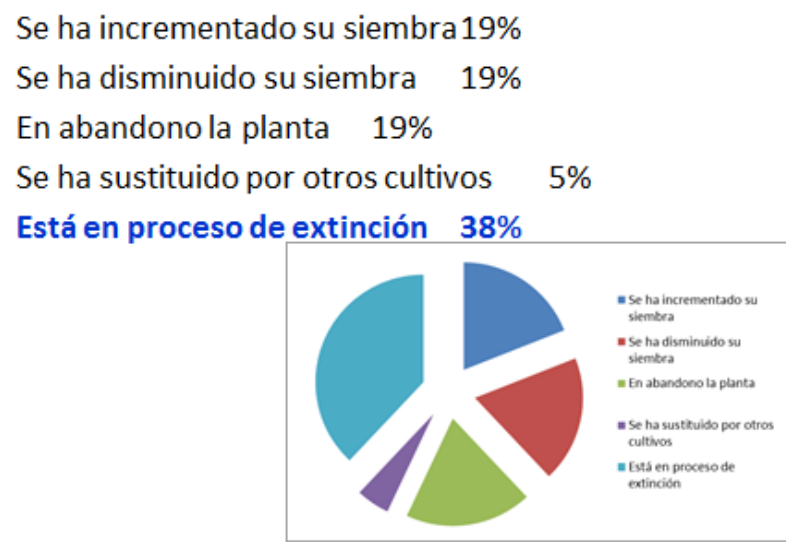

Figura 7. Resultado de encuestas sobre la situación de cultivo en la altiplanicie pulquera hidalguense.

Esta ventaja del maguey pulquero no puede desaprovecharse, a diferencia de los árboles frutales o de sombra que tardan muchos años más en desarrollarse el maguey ofrece muchos beneficios en menor tiempo y con la ventaja de que comienza a generar ingresos al productor en menos tiempo. Es una planta que ofrece beneficios a los cultivos anuales y que no tiene problemas para asociarse con cultivos perennes que se adaptan a las condiciones climáticas y edáficas de la Altiplanicie pulquera hidalguense ya que se puede intercalar sin ningún problema con cultivos como la manzana, ciruela, tejocote, pera, durazno, capulín, chabacano y nopal que son cultivos rentables y demandados por el mercado.

El maguey pulquero tiene la ventaja de no competir por el agua con la mayoría de cultivos, al contrario, su propia naturaleza tiende a captar incluso el rocío y la humedad nocturna y de la niebla beneficiando a las plantas adyacentes, por lo tanto el maguey pulquero ofrece más ventajas que otras plantas incluyendo árboles que tardan más tiempo en desarrollarse para elevar la productividad de cultivos anuales y perennes porque comienza a generar ingresos antes de los ocho años de su desarrollo total y por la multiplicidad de beneficios ambientales y económicos que por sí sola genera.

\section{Conclusiones}

El maguey pulquero es una alternativa factible para revertir la crisis medioambiental, resolver los problemas económicos que enfrentan los productores de la Altiplanicie Pulquera, no solo como un monocultivo, sino recreando el sistema agroforestal tradicional que fue creando desde tiempos prehispánicos, en donde se favorece la biodiversidad, que permite el uso racional y conservación de los recursos naturales, logra convivir con las plagas que genera el monocultivo del maguey y la obtención de múltiples beneficios. 
Está probado que el maguey pulquero combinado con cultivos anuales y perennes como los frutales que se adaptan a esta región del estado de Hidalgo es un sistema eficiente y con el cultivo de otros árboles que requieren poca agua, tolerantes a las heladas, constituye el sistema agroforestal tradicional que resulta fundamental para el aprovechamiento y manejo eficiente de los recursos naturales y la producción de una gran cantidad de satisfactores alimenticios y de otro tipo que cubren las necesidades de los campesinos de la altiplanicei pulquera de Hidalgo.

\section{Literatura citada}

Bartra, A. 2009. La gran crisis. Revista Venezolana de Economía y Ciencias Sociales. 15(2):191-202.

Bartra, A. 2017. Vivimos un capitalismo del fin del mundo, suicida. La Jornada. 26 de marzo de 2017. https://www.jornada.com.mx/2017/03/26/politica/007e1pol.

Beer, J.; Harvey, C.; Ibrahim, M.; Harmand, J. M.; Somarriba, E. y Jiménez, F. 2003. Servicios ambientales de los sistemas agroforestales. Agroforestería en las Américas. 10(37-28):8087. https://www.researchgate.net/publication/228916276_Servicios_ambientales_de_los_ sistemas_agroforestales.

Delgado, R. G. 2014. Extractivismo, ecología política y construcción de alternativas en América Latina. ALASRU. Nueva Época. Análisis Latinoamericano del Medio Rural. 8:17-45.

DOF. 1997. Diario Oficial de la Federación. Norma Oficial Mexicana NOM-007-REC-NAT-1997. http://www.profepa.gob.mx/innovaportal/file/3306/1/nom-007-semarnat-1997.pdf.

García, M. P. C.; Larqué-Saavedra, J.; Eguiarte, L. E. y Zizumbo, V. D. 2007. El futuro de lo ancestral. http://www2.inecc.gob.mx/publicaciones2/libros/537/unamirada.pdf.

Giraldo, O. F. 2014. Utopías en la era de la supervivencia: una interpretación del buen vivir. Editorial Itaca. Departamento de Sociología Rural-Universidad Autónoma Chapingo (UACH). Chapingo, Estado de México. 220 p.

Granados-Sánchez, D. 1993. Los agaves en México. Primera Edición. Universidad Autónoma Chapingo (UACH). Estado de México. 91-97 pp.

Hernández, F. 1959. Historia natural de las plantas de la Nueva España. Universidad Nacional Autónoma de México. Tomo II. México. Prensa Universitaria.

Iglesias, J. M. 1999. Pastos y forrajes. Sistemas de producción agroforestales. Conceptos generales y definiciones. 22:(4)287-305. https://payfo.ihatuey.cu/index.php?journal=pasto\&page= article\&op=view\&path\%5B $\% 5 \mathrm{D}=961$.

INAFED. 2018. Enciclopedia de los municipios y delegaciones de México. Estado de Hidalgo. Regionalización.http://www.inafed.gob.mx/work/enciclopedia/EMM13hidalgo/regionaliz acion.html.

Moncada de la F, J.; Anaya, G. M.; Ortiz, S. C. y Sánchez, G. P. 2013. Suelo. Protejamos el suelo que nos da vida. Colegio de Postgraduados. Texcoco, Estado de México. Folleto técnico.

Narváez, S. A. U. 2015. El maguey pulquero y su aprovechamiento en el ejido de San José de Los Molinos, municipio de Perote, Veracruz. Tesis de maestría en ciencias. Montecillo, México. Colegio de Postgraduados.

Notimex. 2018. Maguey mezcalero ayuda a recargar mantos acuíferos. https://www.posta.com.mx/buena-vida/maguey-mezcalero-ayuda-recargar-mantosacuiferos.

Rivera, V. J. J. 1990. El uso del maguey (Agave sp.) en la conservación del suelo. Tesis de maestría en ciencias. Colegio de Posgraduados. 
Roldán C. E. I. 2017. La planeación pública regional en Hidalgo. El Colegio del Estado de Hidalgo. Roldán, C. E. I. 2015. Organización económica y desarrollo regional del estado de Hidalgo: pasado y presente. El Colegio del estado de Hidalgo.

Ruvalcaba-Mercado, J. 1983. El maguey manso. Historia y presente de Epazoyucan, Hidalgo. Primera Edición. Universidad Autónoma Chapingo (UACH). Texcoco, Estado de México. $122 \mathrm{p}$.

Sifupro.org.mx. 2013. Cultivo del maguey, establecimiento y manejo. http://siproduce.sifupro.org.mx/seguimiento/archivero/29/2013/trimestrales/anexo_21645-2013-11-1.pdf. 